\section{J.-François Lacronique}

Chef du département de santé publique. Faculté de médecine Paris XII.

\title{
Calculs vésiculaires asymptomatiques : l'arbre de décision de la question
}

La question à laquelle viennent de répondre D. Franco et D. Dhumeaux peut être par ailleurs illustrée sous une forme graphique simple entrant dans le cadre de l'analyse de la décision clinique. C'est l'exercice auquel s'est livré 7.-F. Lacronique à partir d'une étude américaine (voir réf. 1). REFERENCES

I. Ransohoff D F, Gracie W A, Wolfenson L B Neuhauser D. Prophylactic cholecystectomy or expectant management for silent gallstones. A decision analysis to assess survival. Ann Int Med 1983; $99:$ 199-204.

2. Tan E G C, Warren K W. Disease of the gallbladder and the bile ducts. In: Schiff L, Schiff E R, eds. Disease of the liver. 5 th ed. Philadelphia; Lippincott J B, 1982: 1 507-59.

3. Schoenfield L J. Disease of the gallbladder and the bile ducts. In Isselbacher $\mathrm{K}$. J, Adams $\mathrm{R} \mathrm{A}$, Braunwald E, Petersdorfr G, Wilson J D, eds. Harrison's Principles of internal Medicine. 9 th ed. New York: McGraw-Hill; 1980: 1489-98.

4. Chigot J. P. Le risque opératoire dans la lithiase biliaire : à propos de 5433 interventions. Sem Hop Paris 1981; 57: $1311-9$.

5. Spiro H M. Clinical Gastroenterology. 2nd ed. New York: MacMillan Publishing Co., Inc; 1977: 926.

\section{ADRESSE}

J. F. Lacronique : Faculté de médecine de Cré-

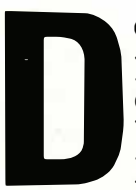
eux attitudes sont donc possibles, schématiquement, face à la lithiase biliaire asymptomatique : la chirurgie ou la surveillance médicale. Les indications de l'une ou de l'autre reposent souvent sur l'âge, quoique certains auteurs $[2,3,5]$ préconisent la cholécystectomie prophylactique systématique. Les arguments en faveur de cette dernière attitude sont simples : elle évite les inconvénients de toutes les complications éventuelles de la maladie, en particulier la cholécystite aiguë, qui imposera elle-même l'intervention dans des conditions plus difficiles.

Mais la chirurgie n'est pas anodine : proposée systématiquement devant toute découverte fortuite de calculs, elle possède ses risques propres, auxquels seront exposés des individus qui n'auraient jamais souffert de leur lithiase.

Comment comparer ces deux "stratégies "? Un essai thérapeutique de type classique est difficile à organiser, pour des raisons éthiques et pratiques. C'est à ce type de questions que l'analyse de la décision clinique est susceptible d'apporter les réponses les plus utiles. Son principe est identique à celui des essais cliniques : il permet une comparaison quantitative des conséquences des deux attitudes, en utilisant pour Cela les mêmes bases décisionnelles que celles utilisées dans la pratique.

Le substrat graphique de cette méthode est l'arbre de décision. Mais il est important de souligner que le terme "arbre de décision" est le plus souvent utilisé en France pour illustrer une démarche par éta-. pes purement logiques; dans cet exemple, emprunté à une équipe américaine [I], il s'agit d'un arbre de décision probabiliste, destiné non pas à guider une démarche, mais au contraire à l'expliciter à l'aide de l'information disponible. Le graphisme de l'arbre de décision est désormais bien codifié : il commence par l'identification du problème à étudier, sous la forme d'une question qui pose un problème de décision humaine : faut-il ou non opérer?

Cette question est figurée sous la forme d'un symbole carré.

Les étapes subséquentes permettent de faire intervenir les conséquences de chaque branche de l'alternative, qui peuvent être soit des "états " constatés, ayant chacun sa probabilité d'apparition, soit d'autres décisions humaines. Les "états" constatés ne sont pas sous dépendance décisionnelle, et sont figurés par un symbole arrondi, matérialisant une fourche aléatoire. $O$

La lecture d'un arbre de décision est habituellement facile : si la décision est celle de la cholécystectomie prophylactique, la première conséquence à apparaître est celle de la mortalité opératoire. Cette première étape étant surmontée, chaque individu est exposé aux autres causes de mortalité, qui s'appliquent à chaque année suivante.

Si la décision est celle de la surveillance médicale, toutes les causes éventuelles de mortalité doivent être comptabilisées. Il s'ajoutera ensuite les possibles complications liées à la présence d'une vésicule lithiasique : crises douloureuses, cholécystite aiguë surtout.

La décision d'intervenir peut apparaitre à n'importe quel moment, avec son éventualité de décès opéra- 


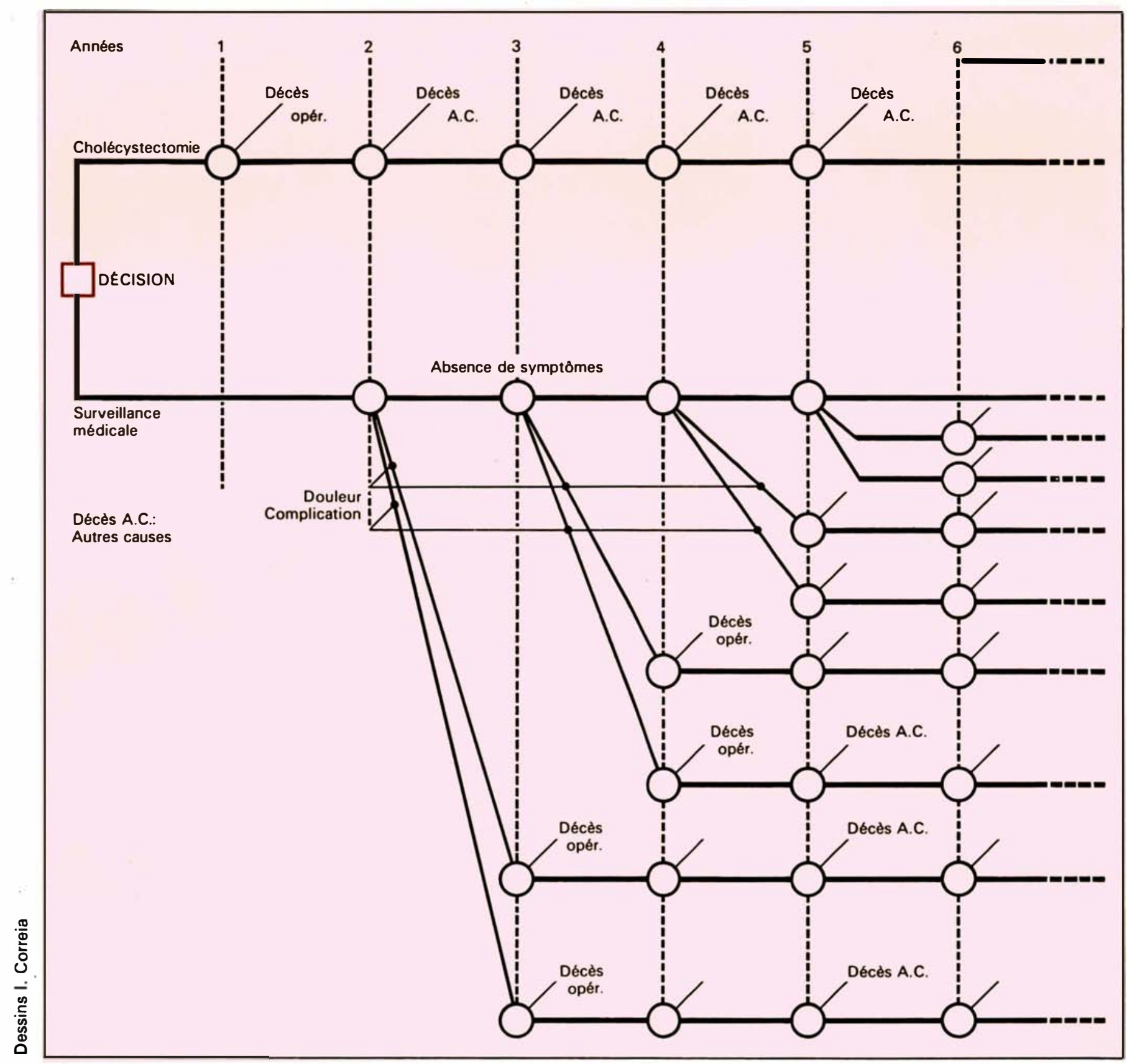

toire propre, qui sera évidemment différente de celle qui était prise en compte dans la première branche. Au total, l'arbre de décision se dessine sous la forme de la figure 1 . L'arbre de décision n'a aucune valeur s'il ne permet pas d'y associer des données quantitatives. Celles-ci sont d'une part les enjeux correspondant à toutes les branches terminales (ici, l'espérance de vie) et d'autre part, les probabilités d'apparition de chaque événement aléatoire.

Les valeurs correspondant à chaque $\mathrm{m} / \mathrm{s}$ avril 85 taux de mortalité opératoire différent beaucoup selon l'âge et le sexe, et selon l'état biliaire antérieur du malade [4]. Ces valeurs sont indiquées sous la forme du tableau I (voir p. suivante). Elles ont été calculées d'après une compilation de la littérature disponible, comme on le fait habituellement dans la pratique clinique, et peuvent être différentes selon les pays. Les valeurs qui figurent ici ont été extraites de la littérature américaine par les auteurs de l'étude de référence.

Une mention particulière doit être
Figure 1. D'après Ransohoff, et al. (réf. 1).

La décision est figurée sous la forme d'un symbole $\square$. Les éventualités aléatoires sont figurées sous la forme d'un symbole O. On a pris ici en compte les décès directement en liaison avec l' acte chirurgical, et les décès dus à d'autres cause (A.C.), puisque l'unité de comparaison choisie est la mortalité. Ce modèle général permet d'appliquer différents types d'hypothèses épidémiologiques, observées ou théoriques. 
faite de la méthode utilisée pour obtenir les probabilités de décès en cas d'intervention survenant après complication biliaire : différentes études ont montré que le risque était multiplié par 4 , par rapport à l'intervention effectuée "à froid ". Les deux lignes de probabilités " après complication biliaire" s'obtiennent donc simplement en multipliant par un facteur 4 les valeurs observées lors d'une intervention systématique, quel que soit l'âge.

La méthode de calcul utilisée en analyse de la décision est simple dans son principe : elle consiste à multiplier chaque "enjeu terminal" (ici, l'espérance de vie), par sa probabilité propre d'apparition.

Pour illustrer ce calcul, imaginons une fourche hypothétique correspondant à "l'état" anatomapathologique d'une vésicule opérée dans un tableau subaigu. Estimons que les valeurs de mortalité soient respectivement de 3,35 et $234 \%$ pour une intervention sur une vésicule qui peut être saine, inflammatoire ou infectée. Les valeurs d'espérance de vie, en années, pour des cohortes de I 000 personnes seront donc de 997,965 et 766 . Ces valeurs seront multipliées par leur fréquence d'observation : imaginons, pour l'exercice, que celles-ci soient respectivement de 70,25 , et $5 \%$ pour chacun des états de la vésicule. (On note que la somme des probabilités pour chaque fourche aléatoire doit être égale à I).

La valeur pondérée de la branche sera donc de :

$997 \times 0,7+965 \times 0,25$

$+766 \times 0,05=977,45$ années.

Un calcul identique sera fait pour chaque branche, de manière à permettre des comparaisons. Les valeurs pondérées obtenues pour chaque fourche aléatoire sont combinées progressivement, fourche par fourche, en parcourant l'arbre de manière récurrente, c'est-à-dire depuis les branches terminales vers le tronc principal.

Le principal intérêt de l'analyse de la décision ne réside pas tant dans les résultats bruts qu'elle permet d'obtenir, mais dans l'étude des variations observées dans le résultat final, lorsqu'on fait varier différents éléments sensibles du modèle. C'est l'" analyse de sensitivité ". Ici, le modèle a été appliqué à différents types de malades d'ages différents.

Ces résultats apparaissent, sous une forme graphique, dans la figure 2, qui montre le nombre respectif de décès consécutifs à l'intervention chirurgicale, (sous la forme d'un trait rouge), et les décès survenant au cours de la surveillance médicale (sous la forme d'une aire hachurée), qui s'étend jusqu'à la fin de la vie.

Dans la figure supérieure, correspondant à 100000 hommes de 50 ans, le nombre de décès observés au décours de la cholécystectomie prophylactique est de 540, comparé à 383 survenant sous surveillance médicale.

Dans la figure inférieure, correspondant à 100000 hommes de 30 ans, l'attitude chirurgicale prophylactique " coûte " 107 décès, et la surveillance médicale 292 .

Chez l'homme de 50 ans, l'intervention chirurgicale provoque indiscutablement plus de décès que la surveillance médicale. Chez l'homme de 30 ans, au contraire, l'interven- tion parait avantageuse, à cause de la faible mortalité opératoire dans cette tranche d'âge. Mais si l'on tient compte de l'espérance de vie potentielle à chaque âge, cet avantage disparait, et l'intervention chirurgicale systématique fait perdre dans tous les cas, quelques jours de vie, par rapport à la stratégie d'observation : 18 jours chez l'homme de 50 ans, 4 chez l'homme de 30 ans, 12 jours chez la femme de 50 ans, I jour chez la femme de 30 ans.

L'analyse de la décision n'est utilisable que pour servir de support à une discussionn et non pour se substituer au raisonnement clinique. Mais les modèles qui sont construits selon cette méthode, comme les chiffres qui leur sont attachés, reflètent souvent les bases du raisonnement humain, avec leurs faiblesses et leur caractère incertain.

Dans le modèle présenté ici, les causes de décès prises en compte ont été volontairement limitées. On n'a pas tenu compte, par exemple, de la probabilité de cancérisation d'une vésicule respectée par le traitement médical, ni d'autres complications rares. Les auteurs en discutent le rôle possible, non sans estimer qu'il est improbable qu'il modifie substantiellement la conclusion d'ensemble.

Celle-ci est cohérente avec celle des experts français; l'analyse de la décision permet ainsi de confirmer quantitativement un jugement que le raisonnement laissait anticiper : l'intervention chirurgicale à titre prophylactique n'est pas justifiée systématiquement dans la lithiase asymptomatique

Tableau 1

MORTALITÉ OPÉRATOIRE APRĖS CHOLÉCYSTECTOMIE POUR 100 OPÉRÉS

\begin{tabular}{|c|c|c|c|c|c|c|c|c|}
\hline \multirow{2}{*}{ circonstances de l'intervention } & \multirow[t]{2}{*}{ Sexe } & \multicolumn{7}{|c|}{ Mortalité opératoire par tranche d'àge } \\
\hline & & 30 & 40 & 50 & 60 & 70 & 80 & 90 \\
\hline \multirow{2}{*}{$\begin{array}{l}\text { intervention prophylactique } \\
\text { ou de confort (douleur...) }\end{array}$} & M & 0,107 & 0,240 & 0,540 & 1,215 & 2,734 & 6,151 & 13,840 \\
\hline & $F$ & 0,053 & $0,1 \cdot 20$ & 0,270 & 0,608 & 1,367 & 3,075 & 6,920 \\
\hline \multirow{2}{*}{ intervention après complication } & M & 0,427 & 0,960 & 2,160 & 4,860 & 10,935 & 24,604 & 55,358 \\
\hline & $F$ & 0,213 & 0,480 & 1,080 & 2,430 & 5,468 & 12,302 & 27,679 \\
\hline
\end{tabular}




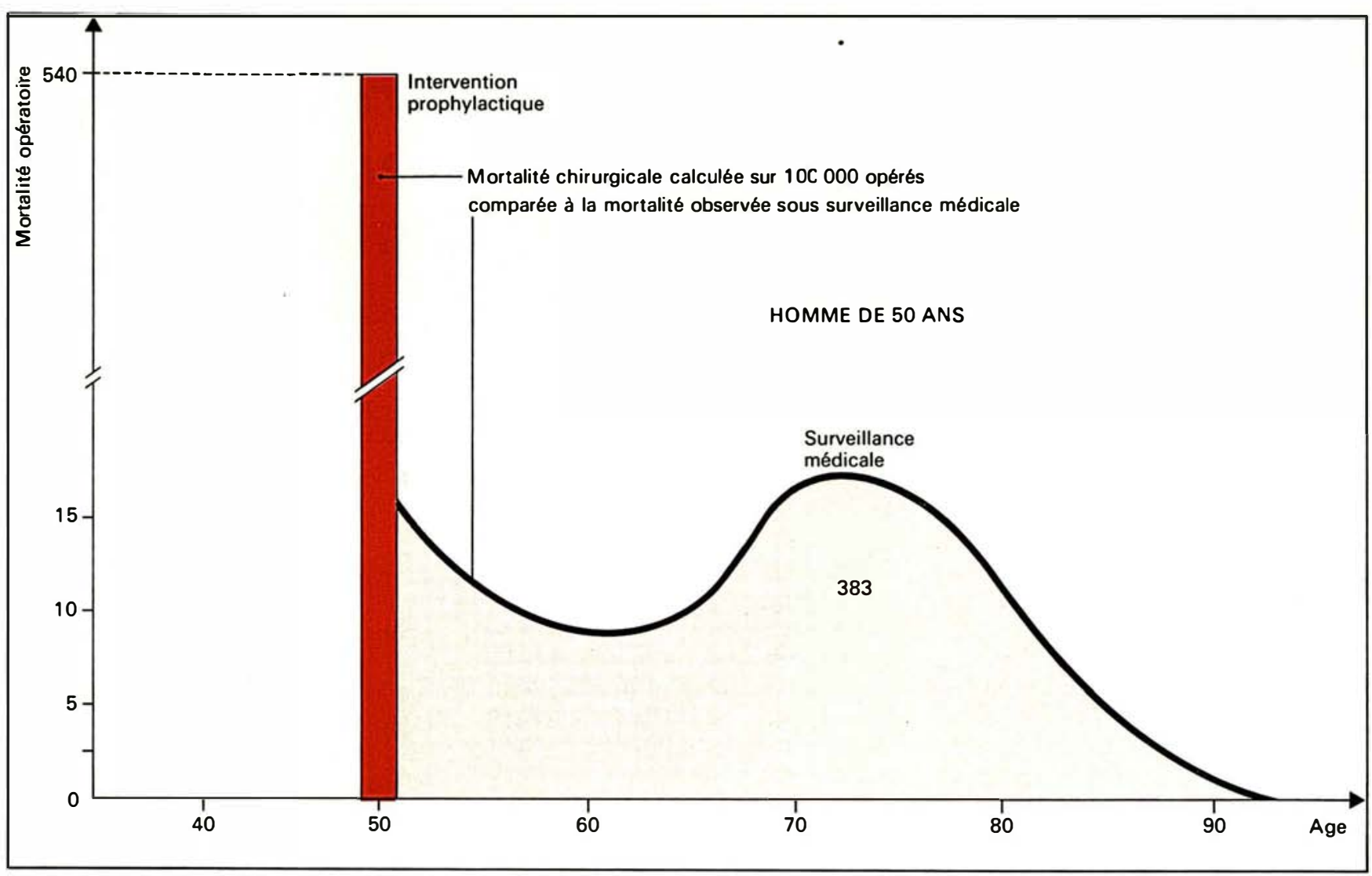

Figure 2. D'après Ransohoff, et al., (réf. 1).

L'application du modèle à deux échantillons de population - hommes de 50 ans et hommes de 30 ans - montrent des variations de résultats théoriques ou tenus de mortalité : dans le groupe des hommes de 50 ans, la mortalité opératoire serait de 540 sur 100.000, à comparer avec une mortalité de 383 sous surveillance médicale. Dans le groupe des hommes de 30 ans, la mortalité opératoire serait de 107 sur I00.000, à comparer avec 292 décès sous surveillance médicale.

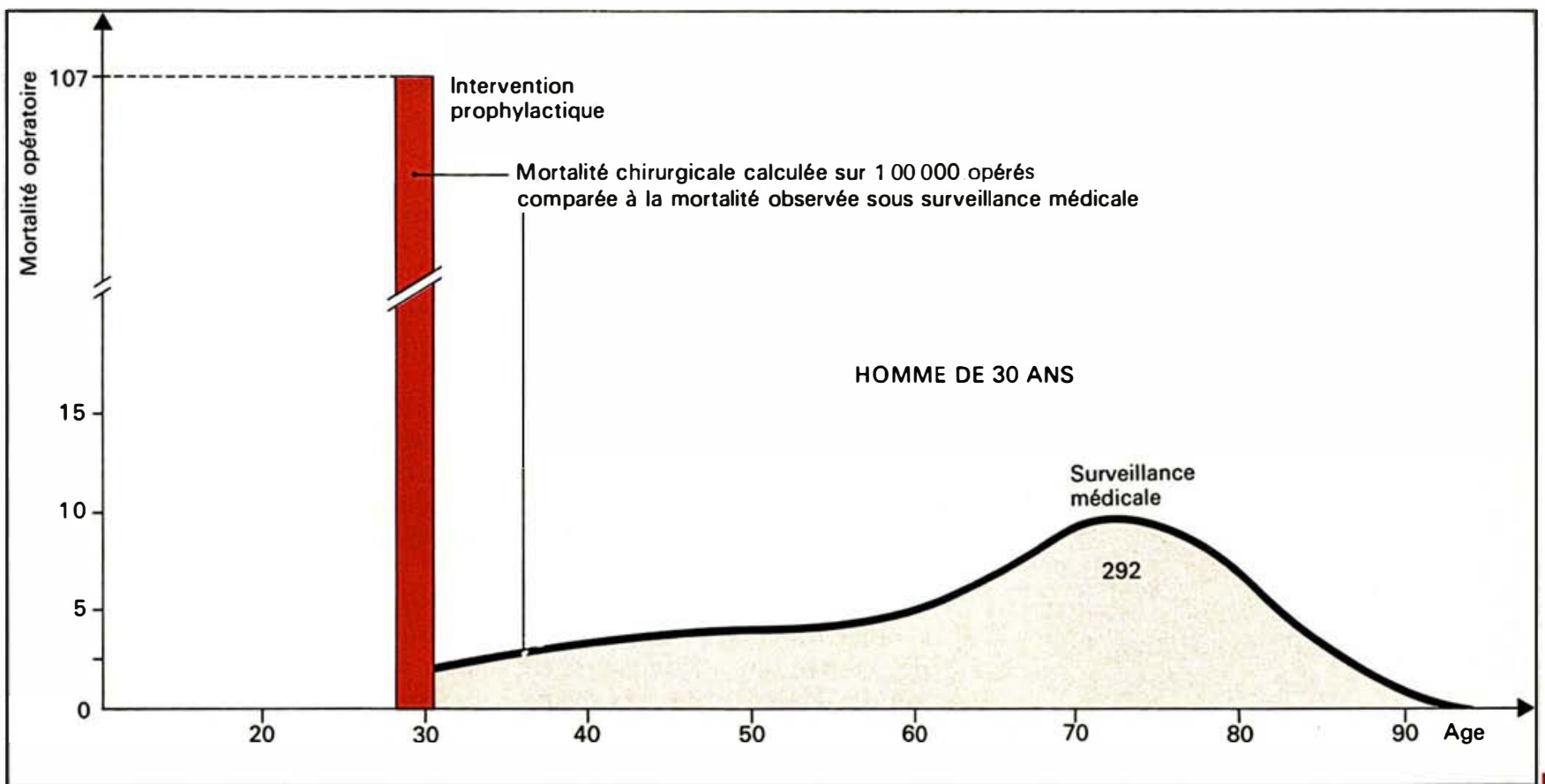

